\title{
Stabilising selection on three chaeta characters in Drosophila melanogaster
}

\author{
N. A. K. Shereif and \\ D. O. F. Skibinski
}

Biomedical and Physiological Research Group, School of Biological Sciences, University College of Swansea, Singleton Park, Swansea SA2 8PP, U.K.

The relationship between yield, a component of fitness related to fertility, and chaeta number is investigated for sternopleural, orbital and ocellar chaetae in Drosophila melanogaster. Central phenotypes have higher yield than extreme for sternopleural and orbital chaeta number in both sexes suggesting the action of stabilising selection. These two characters are highly correlated genetically and phenotypically. Ocellar chaeta number shows no evidence of stabilising selection, nor of significant correlations with the other characters. In males higher yield is associated with faster mating speed.

\section{INTRODUCTION}

Mather (1953) classified selection on quantitative characters into three main types, stabilising, directional and disruptive. Stabilising selection occurs when central phenotypes have a fitness advantage over extremes. It is widely assumed to be the most prevalent type of selection in natural and laboratory populations. Indirect evidence for this comes from the observation that while populations often have stable mean and variance values for particular characters large changes may be achieved by artificial selection. There is controversy about the theoretical consequences of stabilising selection. One view is that it leads to fixation, the other that it leads to polymorphic equilibria (Mather, 1987). Laboratory experiments on artificial stabilising selection on Drosophila melanogaster have produced conflicting results. Although genetic variance usually decreases, environmental variance may either increase or decrease (e.g., Prout, 1962; Scharloo, 1964, 1970; Gibson and Bradley, 1974). A decrease in environmental variance is consistent with the hypothesis of canalising selection (Waddington, 1960).

The number of studies of stabilising selection is relatively small. Stabilising selection has been demonstrated on, for example, body weight in sparrows (Bumpus, 1899), scale and band number in snakes (Camin and Ehrlich, 1958), body size in a lizard (Hecht, 1952), clutch size in birds (Lack,
1954), birth weight in man (Karn and Penrose, 1951; Jayant, 1966) and fin ray number in the guppy (Beardmore and Shami, 1979). Negative results are less widespread, possibly because they are regarded as too inconclusive or uninteresting to merit publication. Thus the true prevalence of stabilising selection is difficult to ascertain.

A number of experiments have shown stabilising selection on sternopleural chaeta number in laboratory populations of $D$. melanogaster. Barnes (1968) demonstrated high fertility of central phenotypes and Kearsey and Barnes (1970) suggest that central phenotypes have high larval viability. This result is interesting as it demonstrates that sternopleural chaeta number cannot itself be the character selected. Individuals with central values for a quantitative character are likely to have higher heterozygosity, at least on an additive model, so this result suggests that sternopleural chaeta number alleles (or alleles in disequilibrium with them) have a pleiotropic and overdominant effect on fitness (see Falconer, 1981 for further discussion). However, Linney et al. (1971) observed stabilising selection on sternopleural chaeta number for larval viability in inbred and highly homozygous lines suggesting that environmental causes of deviation from centrality may also reduce fitness. Stabilising selection on sternopleural and coxal chaeta number in $D$. melanogaster has also been demonstrated using diallele analysis of crosses between inbred 
lines derived from a cage population (Caligari and Mather, 1980; Caligari, 1981).

Here we study stabilising selection with respect to male and female fertility for three characters, sternopleural chaeta number, orbital chaeta number and ocellar chaeta number in flies derived from a cage population of $D$. melanogaster.

\section{MATERIALS AND METHODS}

The flies used in this study were from the Chateau Tahbilk strain of $D$. melanogaster collected from a vineyard at Melbourne, Australia, in 1979. The strain has been maintained since then in a population cage on a standard food medium (Mittler and Bennett, 1962) at $25^{\circ} \mathrm{C}$ and approximately $70 \mathrm{per}$ cent relative humidity under conditions of continuous light.

Flies were raised in $25 \mathrm{~mm} \times 100 \mathrm{~mm}$ glass vials containing $10 \mathrm{ml}$ of food medium. Sternopleural, orbital, and ocellar chaeta number were scored as the sum of the chaetae on the left and right sides of the fly.

The component of fitness studied was the total number of progeny produced per single-pair mating over a defined time period. This component is expected to be highly correlated with fertility, and is referred to as yield.

Two experimental designs were used:

\section{Experiment I}

This design follows that of Barnes (1968). Virgin males and females were obtained from the cage population and separated into chaeta number classes. For each chaeta character the flies were then crossed in all chaeta number combinationsthat is, in diallel fashion. Thus for sternopleural chaeta number individuals in classes 15 to 22 were crossed in all possible ways to give 64 single pair ninatings. For orbital and ocellar chaeta number, individuals in classes 14 to 19 and 5 to 8 respectively were crossed in a similar fashion. Phenotypes with more extreme chaeta numbers were observed for all characters, but were too rare to be included in the crossing programme without having missing cells in the diallel table. Single-pair matings were set up in vials and females allowed to lay for three days. The number of progeny per single pair mating was scored separately for the first three days of emergence and for the remaining period of emergence.

\section{Experiment /I}

Five hundred virgin flies of each sex were obtained from the cage population and distributed randomly into vials by placing one fly of each sex into each vial. These allowed to lay for three days before being scored for their sternopleural, orbital, and ocellar chaeta number. They were then discarded. The total number of progeny emerging from each vial was then recorded. Twenty vials with the highest number of progeny, 20 with the lowest number (excluding infertile pairs giving zero progeny), and 20 vials giving zero progeny, were identified. These three sets of vials give rise to the "high", "low", and "zero" progeny classes respectively. Twenty vials were also chosen at random as a control.

\section{RESULTS}

The mean values of the three chaeta characters over the eight months just prior to the crossing experiments in the cage population are shown in fig. 1. Both sexes showed more or less stable values for all chaeta characters during this period. This suggests that the genetic structure of the population was largely stable. Values of the three characters for the base population experiment from the parents and three progeny of each sex derived from 176 random pair mating scored for the three chaeta characters are given in table 1 . The narrow sense

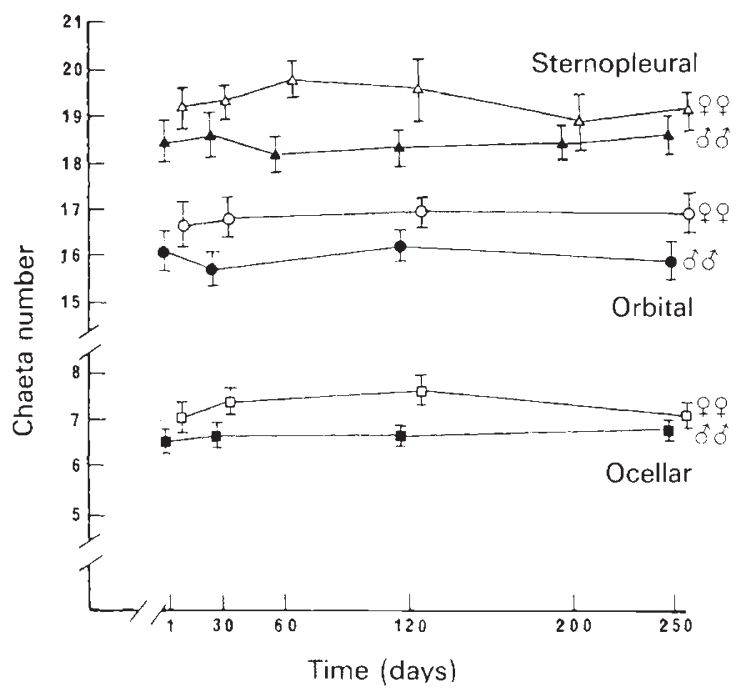

Figure 1 Temporal variation in mean of the three chaeta characters studied in a cage population of Drosophila melanogaster. 50 flies of each sex were used to estimate the mean and standard error. 
Table 1 Means, heritabilities, and phenotypic, genetic, and environmental correlations for three chaeta characters in Drosophila melanogaster. Standard deviations are given for the means and standard errors for the heritabilities and correlations

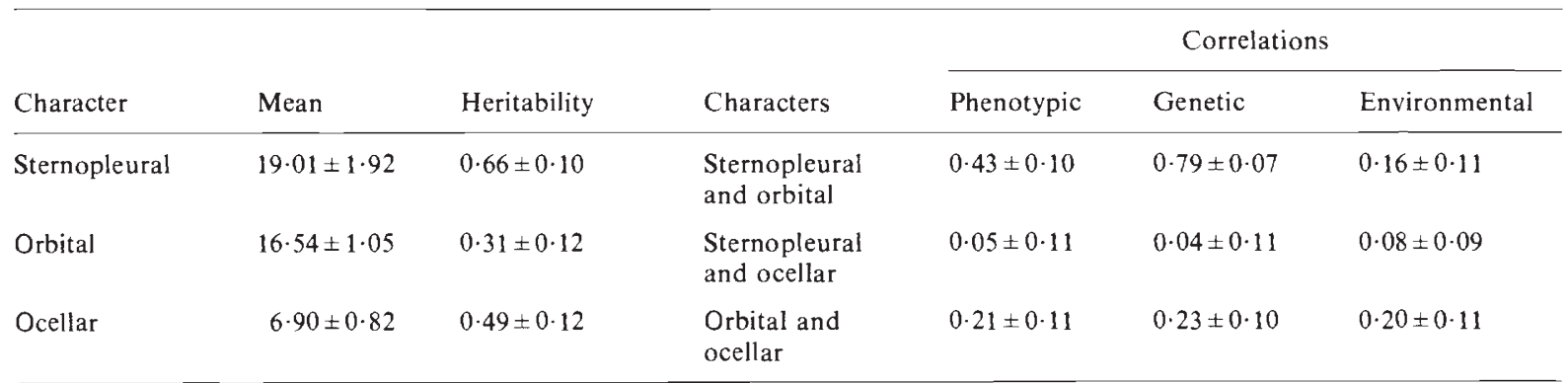

heritability of all characters is within the range expected for these meristic characters in a large outbred cage population. Phenotypic and genetic correlations between sternopleural and orbital chaeta number are high while those involving ocellar chaeta number are much lower (particularly the correlations between ocellar and sternopleural chaeta number).

The yields for the entire period of emergence for each chaeta character are shown for experiment I in figs 2 and 3 . The yields for each chaeta number class are calculated as the means of the separate male and female array mean yield for the diallel matrix.

For each chaeta character the distribution of yield is roughly bell-shaped, with intermediate chaeta numbers having the highest yield. The 18 , 16 and 6 chaeta number classes produce the highest number of progeny for sternopleural, orbital and ocellar chaeta respectively. Deviation from these intermediate values usually results in a reduction in yield. The optimal chaeta classes are close to
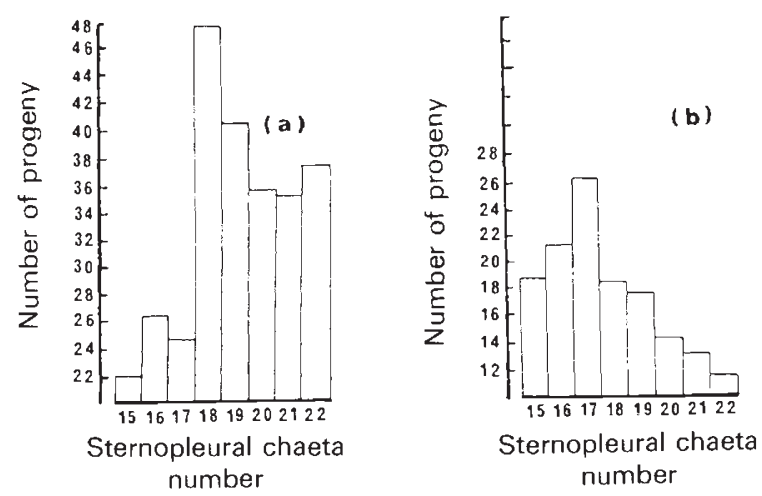

Figure 2 Mean yield for sternopleural chaeta number phenotypes. Number of progeny emerging were counted over three days in (a), and for the remainder of the emergence period in (b). the population means of the characters shown in fig. 1. All three characters are consistent in showing higher values for this component of fitness for central phenotypes.

Variation in yield between different chaeta classes was assessed by analysis of variance. First, a three-way ANOVA was carried out for each character, true dimensions being female chaeta number, male chaeta number and emergence period (early emergents and late emergents). The yield values were square root transformed before analysis. The first order interactions between chaeta number and emergence period assessed against the third order interaction were significant only for sternopleural chaeta number $\left(F_{7,49}=\right.$ 5.9051, $P<0.001$ for male chaeta $\times$ emergence period and $F_{7,49}=3.8871, P<0.01$ for female chaeta $\times$ emergence period). This suggests that the pattern of yield for different sternopleural chaeta number classes differs between early and late emergents. Figs. 2(a) and 2(b) show that for early emergents higher chaeta numbers have greater yield
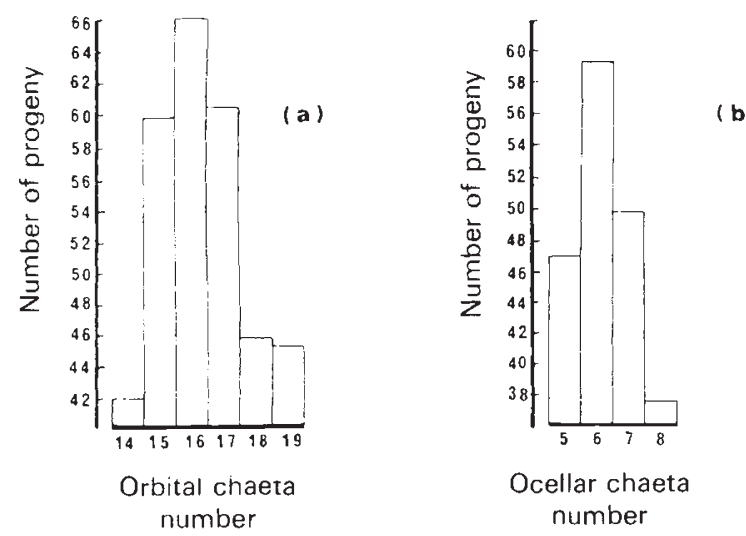

Figure 3 Mean yield for total emergents for orbital chaeta number of phenotypes in (a) and ocellar phenotypes in (b). 
than lower chaeta numbers: for late emergents this difference is reversed. Central phenotypes have higher yield in both distributions but it is noticeable that the modal class for late emergents (17) has very low yields for early emergents. No significant interaction was observed between male and female chaeta number for any of the characters. Yield was significantly greater for the early emergence period for all characters $(65,67$ and 74 per cent of total emergents for sternopleural, orbital and ocellar chaeta respectively). Some results of the analyses of variance are shown in table 2 . Because of the interaction with emergence period for sternopleural chaeta number, separate two-way analyses were carried out for early and late emergents. Analyses were carried out on total emergents for the other two characters. Both male and female chaeta components are significant for late emergents and the male component is significant for early emergents for sternopleural chaeta number. No other components are sig- nificant although the male component for orbital chaeta number is close to significance. The nature of the interaction between chaeta number and emergence period is not substantially different between males and females. In both sexes higher sternopleural chaeta number phenotypes have higher yield amongst early emergents while lower sternopleural chaeta number phenotypes have higher yield amongst late emergents. The differences between the sexes in table 2 appear to be the result of a difference in the magnitude of this interaction effect.

An additional experiment assessed the possible relationship of mating speed and yield for the early emergence period. Sixty pairs of virgin flies were set up in vials and the time until the commencement of mating was recorded for each pair. The 10 fastest mating and 10 slowest mating pairs were scored for sternopleural chaeta number and progeny yield assessed over the first three days of emergence. For males the mean chaeta values were

Table 2 Results of analyses of variance of yield for sternopleural, orbital and ocellar chaeta number in Drosophila melanogaster

\begin{tabular}{|c|c|c|c|c|c|}
\hline & d.f. & M.S. & $F$ & $\begin{array}{l}\text { Variance } \\
\text { component }\end{array}$ & $\%$ of total \\
\hline \multicolumn{6}{|l|}{$\begin{array}{l}\text { Sternopleural chaetae } \\
\text { (late emergents) }\end{array}$} \\
\hline Males & 7 & 2.69 & $2 \cdot 27^{*}$ & $0 \cdot 19$ & 10 \\
\hline Females & 7 & $4 \cdot 70$ & $3 \cdot 96^{* *}$ & 0.44 & 24 \\
\hline Males × females (Error) & 49 & $1 \cdot 19$ & & $1 \cdot 19$ & 65 \\
\hline \multicolumn{6}{|l|}{$\begin{array}{l}\text { Sternopleural chaetae } \\
\text { (early emergents) }\end{array}$} \\
\hline Males & 7 & $7 \cdot 98$ & $4.95^{* * *}$ & 0.80 & 30 \\
\hline Females & 7 & $3 \cdot 52$ & $2 \cdot 18 \mathrm{~ns}$ & $0 \cdot 24$ & 9 \\
\hline Males $\times$ females (Error) & 49 & $1 \cdot 61$ & & $1 \cdot 61$ & 61 \\
\hline \multicolumn{6}{|l|}{$\begin{array}{l}\text { Orbital chaetae } \\
\text { (total emergents) }\end{array}$} \\
\hline Males & 5 & $5 \cdot 45$ & $2 \cdot 57 \dagger$ & 0.67 & 22 \\
\hline Females & 5 & $3 \cdot 05$ & $1 \cdot 44 \mathrm{~ns}$ & $0 \cdot 19$ & 6 \\
\hline Males $\times$ females (Errors) & 25 & $2 \cdot 12$ & & $2 \cdot 11$ & 71 \\
\hline \multicolumn{6}{|l|}{$\begin{array}{l}\text { Ocellar chaetae } \\
\text { (total emergents) }\end{array}$} \\
\hline Males & 3 & $1 \cdot 41$ & $1 \cdot 52 \mathrm{~ns}$ & $0 \cdot 16$ & 10 \\
\hline Females & 3 & $2 \cdot 31$ & $2 \cdot 49 \mathrm{~ns}$ & 0.46 & 30 \\
\hline Males $\times$ females (Error) & 9 & 0.93 & & 0.93 & 60 \\
\hline \multicolumn{6}{|l|}{$\begin{array}{l}\text { Sternopleural chaetae } \\
\text { (early emergents) }\end{array}$} \\
\hline Males & 6 & $3 \cdot 86$ & $11 \cdot 31^{* * *}$ & $0 \cdot 15$ & 16 \\
\hline Females & 6 & $3 \cdot 22$ & $9 \cdot 43^{* * *}$ & 0.12 & 13 \\
\hline Interaction & 36 & $1 \cdot 19$ & $3 \cdot 48^{* * *}$ & 0.28 & 32 \\
\hline Error & 98 & 0.34 & & 0.34 & 38 \\
\hline
\end{tabular}

\footnotetext{
$* P<0.05$.

$* * P<0.01$.

*** $P<0.001$.

† Close to $P<0.05$.
} 
$19 \cdot 40 \pm 0 \cdot 40$ (standard error) and $17 \cdot 40 \pm 0 \cdot 58$ for the fastest and slowest groups respectively; the values for females were $19 \cdot 60 \pm 0.76$ and $19 \cdot 70 \pm$ $0 \cdot 52$. Only the male difference is significant $t=$ $2 \cdot 83, P<0 \cdot 02$. The mean yields for the fastest and slowest groups were $40 \cdot 40 \pm 5 \cdot 27$ (standard error) and $26 \cdot 10 \pm 4 \cdot 19$ respectively $(t=2 \cdot 12, P<0 \cdot 05)$. No significant differences were found between the variances of sternopleural chaeta number. It thus appears that the fastest mating males have high sternopleural chaeta number and yield, a result consistent with the nature of the interaction between male chaeta number and emergence period. No such result is found for females which is consistent with the non-significant and smaller effect of female chaeta number on early emergents (table 2).

Experiment I was repeated for sternopleural chaeta number over a range of 16-22 chaeta and with three replicates for each of the $7 \times 7$ possible chaeta crosses. Only early emergents were counted. This design enables the main and interaction components of the ANOVA to be tested against a true error arising from replicate differences. The results are also shown in table 2 and the array mean yields are shown for sexes combined in fig. 4. They corroborate those obtained earlier in showing a higher yield for central than for more extreme phenotypes. The male $\times$ female interaction is significant and arises because there is a more flattened yield distribution for males than females. This significant interaction might not have been detected in the non-replicated experiments because of inflation of the second order interaction mean square used as error in the three way ANOVA with a real interaction component. The partitioning of the variance components in table 2 suggest that $30-40$ per cent of the total variation for all characters may be due to chaeta effects. There is, however, some variation in the relative magnitude of the male and female effects. For example for sterno-

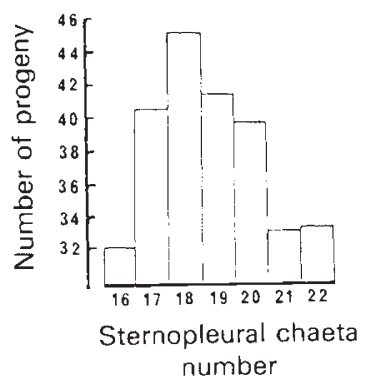

Figure 4 Mean yield of early emergents for different sternopleural chaeta number phenotypes. pleural chaeta number greater variation is attributable to males for early emergents and to females for late emergents.

In experiment I, chaeta number can be regarded as the independent and yield the dependent variable. In experiment II this relationship is reversed as parent flies were selected according to the number of progeny they produced. As the total number of crosses used was much greater than in Experiment $I$ the chance of obtaining significant results might be increased. Also, because the same number of crosses were set up for each character the effects on yield of different characters will be more comparable. Low yield phenotypes are predicted to occur more often at the extremes of the distribution whereas high yield will be more central. As a result, the variance of chaeta number will be greater for low than high yield classes. Similarly the variance of the control class might be expected to be intermediate between the variances of the high and low yield classes. A high variance would also be expected for the zero progeny class.

The means and phenotypic variances of the chaeta characters are given in table 3 for the four classes of progeny together with $F$ ratio values for variance comparisons. Data for male and female parents are tabulated separately. Within sexes the means for the four classes are quite similar. However, some large differences are apparent between variances. For the low, high and control classes the differences are in line with predictions for sternopleural and orbital chaeta number. Most notable is the high variance of the low compared with high class. For ocellar chaeta number the differences between the variances are small. For all characters the variance value for the zero class is lower than that for the low class and not consistently different from the control. The chaeta distributions for the high and low classes are shown in fig. 5. The greater spread of the chaeta distribution for the low class is apparent for sternopleural and orbital chaeta number but not for ocellar chaeta number.

\section{DISCUSSION}

These results corroborate those of Barnes (1968) and others in showing higher values for a component of fitness related to fertility for central than extreme sternopleural chaeta number. There is also evidence of stabilising selection for orbital chaeta number but not for ocellar chaeta number. If stabilising selection is operating on ocellar chaeta 

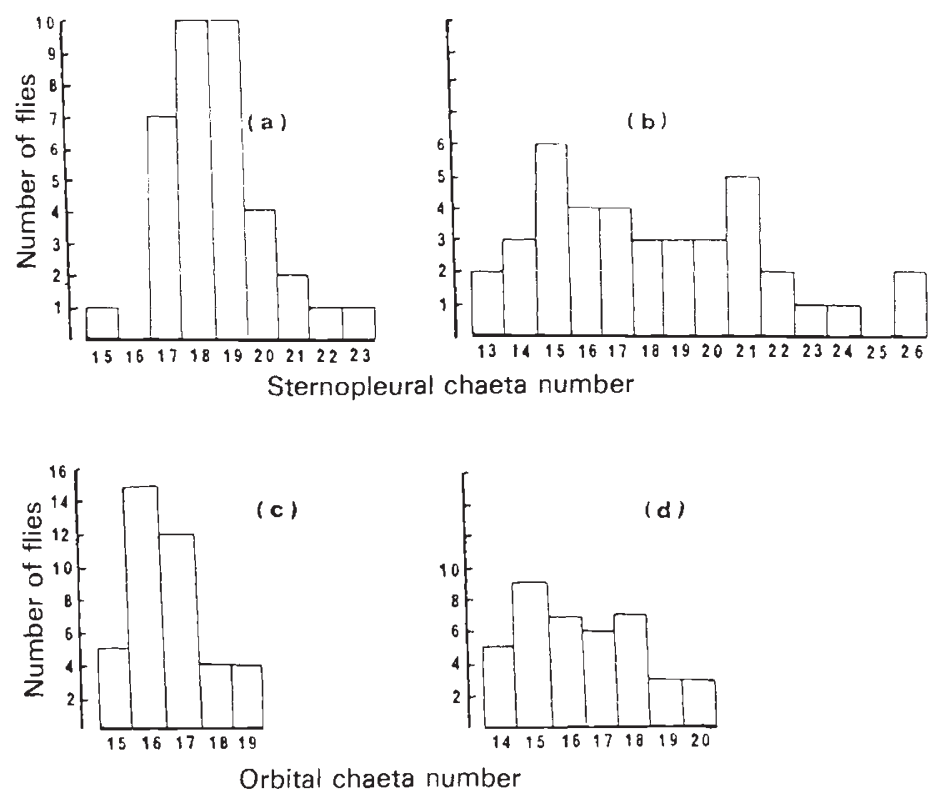

Orbital chaeta number
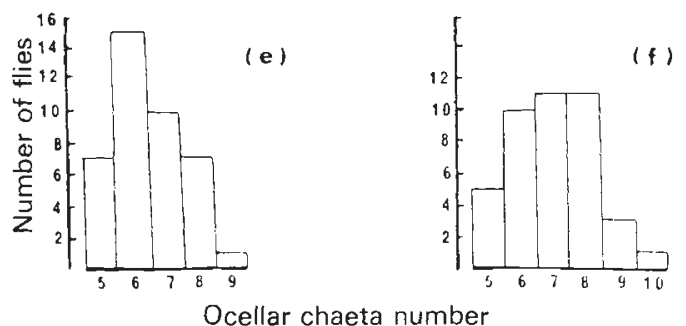

Figure 5 Distribution of chaeta number for high and low yield progeny classes. (a) high sternopleural chaeta yield, (b) low sternopleural chaeta yield, (c) high orbital chaeta yield, (d) low orbital chaeta yield, (e) high ocellar chaeta yield, (f) low ocellar chaeta yield.

number with respect to the yield component of fitness the intensity of selection must be much lower than for the other two characters.

The differences between the characters are not related to differences in their heritability values. However, sternopleural chaeta and orbital chaeta number two characters that are highly correlated genetically and phenotypically show similar patterns of stabilising selection. Ocellar chaeta number, an uncorrelated character, shows less evidence of stabilising selection. The choice of sternopleural chaeta number might hence have been particularly fortuitous. If central chaeta genotypes do have a pleiotropic effect on fitness by constraining development along some optimal path the magnitude of this effect must vary between characters.

Barnes (1968) did not distinguish between male and female effects on fertility. We find that the association of fertility and sternopleural chaeta number occurs for both sexes and also that the pattern of chaeta effects differs between early and late emergents. Since the laying period and early emergence period are both three days long, the differential effect of chaeta number on late emergence is probably related to variation in the time of egg to adult development. The high yield of high sternopleural chaeta number phenotypes amongst the early emergents appears to be associated with male mating speed. This is interesting in view of the emphasis placed on male mating success by exponents of sexual selection.

This study and others demonstrate the association of sternopleural chaeta number with several components of fitness.

This association could be explained either by a pleiotropic effect on fitness of chaeta factors or by the linkage in disequilibrium of chaeta factors to alleles at other polymorphic fitness loci. It is 
Table 3 Mean and phenotypic variance of chaeta characters for samples of 20 flies selected according to yield for the total emergence period

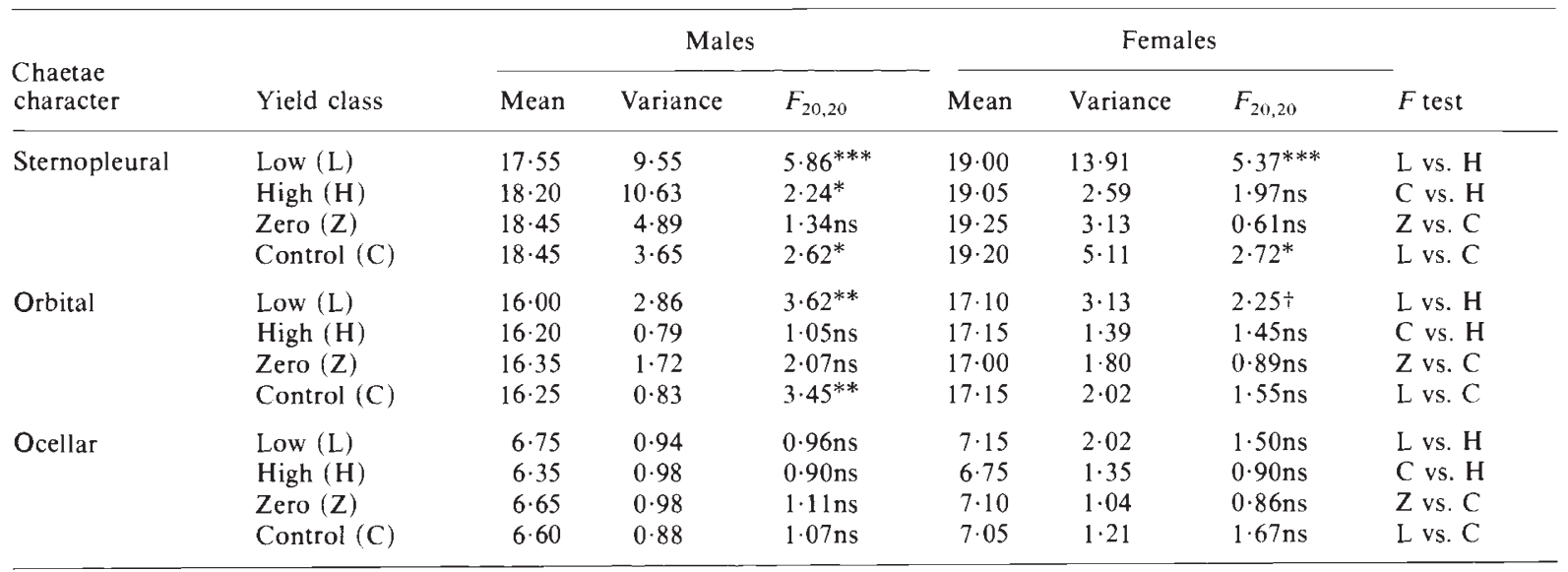

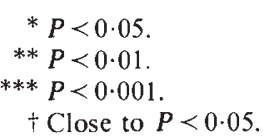

unfortunately not yet possible to distinguish between these two alternative hypotheses. Both are consistent with overdominance at fitness loci and the maintenance of genetic variation for chaeta number by heterozygous advantage.

Epistasis between fitness loci might also be involved in maintaining chaeta variation. It is now of great interest to investigate stabilising selection simultaneously for a range of meristic or continuous characters with respect to several components of fitness.

Acknowledgements We thank Professor J. A. Beardmore for helpful comments and Mrs M. Warren for technical assistance.

\section{REFERENCES}

BARNES, B. W. 1968. Stabilising selection in Drosophila melanogaster. Heredity, 23, 433-442.

BEARDMORE, J. A. AND SHAM 1, S. A. 1979. Heterozygosity and the optimum phenotype under stabilising selection. Aquilo. ser. Zool., 20, 100-110.

BUMPUS, H. C. 1899. The elimination of the unfit as illustrated by the introduced sparrow. Biol. Woods. Hole, 11, 209-226.

CALIGARI, P. D. S. 1981 . The selectively optimal phenotypes of the coxal chaeta in Drosophila melanogaster. Heredity, 47, $79-85$.

CAligari, P. D. S. AND MATHER, K. 1980. Dominance, allele frequency and selection in a population of Drosophila melanogaster. Proc. Royl. Soc. Lond. B., 208, 163-187.

CAMIN, J. H. AND EHRLICH, P. R. 1958. Natural selection in water snakes (Natrix sipedon, L.) on islands in Lake Erie. Evolution, 12, 504-511.

FALCONER, D. S. 1981. Introduction to Quantitative Genetics, 2nd ed. Longman, New York.
GIBSON, J. B. AND BRADLEY, B. P. 1974. Stabilising selection in constant and fluctuating environments. Heredity, 33, 293-302.

HECHT, M. K. 1952. Natural selection in the lizard genus Aristelliger. Evolution, 6, 112-124.

JAYANT, K. 1966. Birth weight and survival: A hospital survey repeat after 15 years. Ann. Hum. Genet. (Lond.), 29, 367375.

KARN, M. N. AND PENROSE, L. S. 1951. Birth weight and gestation time in relation to maternal age, parity and infant survival. Ann. Eugen. (Lond.), 16, 147-164.

KEARSEY, M. J. AND BARNES, B. W. 1970. Variation for metrical characters in Drosophila populations. II. Natural selection. Heredity, 25, 11-21.

LACK, D. 1954. The evolution of reproductive rates. In Huxley, J., Hardy, A. L. and Ford, E. B. (eds.) Evolution as a Process, pp. 143-156.

LINNEY, R., BARNES, B. W. AND KEARSEY, M. J. 1971. Variations for metrical characters in Drosophila populations. III. The nature of selection. Heredity, 27, 163-174.

MATHER, K. 1953. The genetical structure of populations. Symp. Soc. Exp. Biol., 7, 66-95.

MATHER, K. 1987. Consequences of stabilising selection for polygenic variation. Heredity, 58, 267-277.

MITTLER, S. AND BENNET, J. 1962. A simple food medium that requires no live yeast with the minimum of variables. Dros. Inf. Serv., 36, 131-132.

PROUT, T. 1962. The effects of stabilising selection on the time of development in Drosophila melanogaster. Genet. Res., 3, 364-382.

SCHARLOO, w. 1964. The effects of disruptive and stabilising selection on the expression of a cutibus interruptus mutant in Drosophila. Genetics, 50, 553-562.

SCHARLOO, w. 1970. Stabilising and disruptive selection on a mutant character in Drosophila. III. Polymorphism caused by a developmental switch mechanism. Genetics, 65, 693705.

WADDINGTON, C. H. 1960. Experiments on canalising selection. Genet. Res., 1, 140-150. 